Pacific Journal of Mathematics

SOME ASPECTS OF TORSION

mick Jars 


\title{
SOME ASPECTS OF TORSION
}

\author{
J. P. JANS
}

\begin{abstract}
Using S. E. Dickson's characterization of a torsion class, a class of modules closed under taking factors extensions and arbitrary direct sum, we study torsion classes closed under taking submodules and arbitrary direct products. We show that these classes are in one-to-one correspondence with idempotent two sided ideals of the ring. Finally we investigate the structure of rings $R$ for which the torsion class $\mathscr{T}_{0}=$ $\left\{M \mid \operatorname{Hom}_{R}(M, Q(R))=0, Q(R)\right.$ the minimal injective for $\left.R\right\}$ is closed under taking products.
\end{abstract}

The original purpose of this paper was to show that for certain rings the direct product of torsion modules is again torsion, and by torsion we mean a particular kind of torsion defined in $\S 3$. However, we find that S. E. Dickson [3] has given a set of axioms for torsion theories in Abelian categories and we thought it best to work within his axiomatic system.

In $\S 1$ we summarize the work of Dickson and study, within the context of modules over a ring, torsion theories closed under taking submodules. In $\S 2$, we give a complete characterization of all torsion theories closed under taking submodules and direct products. Finally, in $\S 3$ we show that a fairly wide class of rings enjoy the property that a particular kind of torsion is closed under taking submodules and direct products.

1. Sets of torsion theories. Dickson [3] has introduced a set of axioms for torsion theories in certain abelian categories sufficiently general to include the category $R$. $/ C$ of left modules and homomorphisms over a ring $R$ with identity. His axioms (stated here for ${ }_{R} / l$ ) are as follows.

A torsion theory for ${ }_{R} / l$ is a pair $(\mathscr{T}, \mathscr{F})$ of classes of modules such that:

I. $\mathscr{T}$ and $\mathscr{F}$ have only 0 in common.

II. $\mathscr{T}$ is closed under taking factors and $\mathscr{F}$ is closed under taking submodules.

III. For each $M$ in ${ }_{R}$. $/ /$ there exists a unique submodule $T(M)$ of $M$ such that

$$
0 \rightarrow T(M) \rightarrow M \rightarrow M / T(M) \rightarrow 0
$$

is exact with $T(M) \in \mathscr{T}$ and $M / T(M) \in \mathscr{F}$.

Received July 15, 1964. The author gratefully acknowledge the support of NSF grant GP 809. 
The modules in $\mathscr{F}$ are torsion modules and those in $\mathscr{F}$ are torsion free. $T(M)$ is the unique largest submodule of $M$ in $\mathscr{T}$.

Dickson [3] proved a number of things about torsion theories (in a context more general than the category ${ }_{R} \mathscr{C l}$ ); we state a few of his results. In what follows $(\mathscr{T}, \mathscr{F})$ is a torsion theory for ${ }_{R} \mathscr{C l}$.

A. $\mathscr{T}$ is closed under direct sum and $\mathscr{F}$ is closed under direct product.

B. Both $\mathscr{T}$ and $\mathscr{F}$ are closed under extension.

C. Each class $\mathscr{T}, \mathscr{F}$ uniquely determines the other.

D. Given $\mathscr{T}$ closed under taking factors extension and direct sum, there exists a unique $\mathscr{F}=\left\{F \mid \operatorname{Hom}_{R}(T, F)=0\right.$ all $\left.T \in \mathscr{T}\right\}$ such that $(\mathscr{T}, \mathscr{F})$ is a torsion theory.

$\mathrm{D}^{\prime}$. Given $\mathscr{F}$ closed under taking submodules extensions and direct product there exists a unique class $\mathscr{T}=\left\{T \mid \operatorname{Hom}_{R}(T, F)=0\right.$ all $F \in \mathscr{F}\}$ such that $(\mathscr{T}, \mathscr{F})$ is a torsion theory.

Thus in considering torsion theories it is enough to consider classes $\mathscr{T}$ closed under taking factors extensions and direct sums (or classes $\mathscr{F}$ closed under taking submodules extensions and direct products).

For the rest of this paper we shall only consider torsion theories $(\mathscr{F}, \mathscr{F})$ for ${ }_{a} / \mathrm{l}$ where $\mathscr{T}$ is closed under taking submodules as well. With respect to this property Dickson [3] proved the following $E$. $\mathscr{T}$ is closed under taking submodules if and only if $\mathscr{F}$ is closed under taking minimal injectives.

We shall use the phrase "a torsion theory closed under taking submodules" to describe this situation.

It should be noted that Dickson was working in a context more general than modules over a ring and that we have specialized his results to this case.

In a torsion theory $(\mathscr{F}, \mathscr{F})$ closed under taking submodules, the torsion class $\mathscr{T}$ forms a "strongly complete additive" class in the sense of Walker and Walker [10]; that is, $\mathscr{T}$ is a class closed under taking factors submodules and arbitrary direct sums. Gabriel [6] has shown that there is a one-to-one correspondence between such classes and filters of left ideals of the ring $R$. Consequently, the torsion theories closed under taking submodules form a set.

In the following theorem we connect such a torsion theory with injective modules.

THEOREM 1.1. If $(\mathscr{T}, \mathscr{F})$ is a torsion theory closed under taking submodules then

(1) There exists modules $X_{0} \in \mathscr{T}$ and $Y_{0} \in \mathscr{F}$ such that $\mathscr{F}=$ $\left\{Z \mid \operatorname{Hom}_{R}\left(X_{0}, Z\right)=0\right\}$ and $\mathscr{T}=\left\{W \mid \operatorname{Hom}_{R}\left(W, Y_{0}\right)=0\right\} \quad\left(X_{0}\right.$ and $Y_{0}$ are not unique).

(2) The module $Y_{0}$ can be selected to be injective. 
Proof. Let $\left\{X_{\alpha}\right\}$ be the set of finitely generated modules in $\mathscr{G}$. We say the set we mean, of course, one module from each equivalence class (equivalence under isomorphism) of finitely generated modules. Now since $\mathscr{T}$ is closed under taking submodules, factors and direct sums, it is therefore closed under direct limit. Thus a module is in $\mathscr{T}$ if and only if its finitely generated submodules are in $\mathscr{T}$. Now let $X_{0}=\Sigma_{\alpha} X_{\alpha}$ be the direct sum of the finitely generated modules in

Now form the class $\mathscr{F}^{\prime}=\left\{M \mid \operatorname{Hom}_{R}\left(X_{0}, M\right)=0\right\}$. It is clear that $\operatorname{Hom}_{R}\left(X_{0}, M\right)=0$ if and only if $\operatorname{Hom}_{R}\left(X_{\alpha}, M\right)=0$ for all the modules $X_{\alpha}$. Therefore the class $\mathscr{F}^{\prime}$ contains the class $\mathscr{F}$. We show them equal. Let $M$ be in $\mathscr{F}^{\prime}$ but not in $\mathscr{F}$, then $0 \neq T(M) \leqq M$. But $T(M)$ contains a finitely generated module $(\neq 0)$ isomorphic to one of the $X_{\alpha}$. It follows that $\operatorname{Hom}_{R}\left(X_{\alpha}, T(M)\right), \operatorname{Hom}_{R}\left(X_{\alpha}, M\right)$ and $\operatorname{Hom}_{R}\left(X_{0}, M\right)$ are all nonzero contradicting the assumption that $M$ was not in $\mathscr{F}$. Thus we have shown that $\mathscr{F}=\left\{M \mid \operatorname{Hom}_{R}\left(X_{0}, M\right)=0.\right\}$

Now choose a set of finitely generated modules $\left\{X_{a}^{\prime}\right\}$ one from each isomorphism class not in $T$. Factoring out the $\mathscr{T}$ torsion of each of these we obtain a set of modules $F_{\alpha}$ all in $\mathscr{F}$, where $F_{\alpha}=X_{\alpha}^{\prime} / T\left(X_{\alpha}^{\prime}\right)$.

Let $Y_{0}=Q\left(\pi F_{\alpha}\right)$, the minimal injective containing the product $\pi F_{\alpha}$. Recall that for a module $M, Q(M)$ is injective containing $M$ as a submodule, and for $X \neq 0$ in $Q(M) X \cap M \neq 0$ also. See [4]. The module $\pi F_{\alpha}$ is in $\mathscr{F}$ because $\mathscr{F}$ is closed under products. Also from property $E$ cited above $\mathscr{F}$ is closed under taking minimal injectives, consequently $Y_{0}$ is in $\mathscr{F}$.

Now form the class $\mathscr{T}^{\prime}=\left\{X \mid \operatorname{Hom}_{R}\left(X, Y_{0}\right)=0\right\}$. From the properties of Hom, [9], $\mathscr{T}^{\prime}$ is closed under taking factors, extensions, and direct sums. But the fact that $Y_{0}$ is injective implies that $\mathscr{T}^{\prime}$ is also closed under taking submodules, for if $0 \rightarrow X^{\prime} \rightarrow X$ is exact, then $\operatorname{Hom}_{R}\left(X, Y_{0}\right) \rightarrow \operatorname{Hom}_{R}\left(X^{\prime}, Y_{0}\right) \rightarrow 0$ is also exact. That is, if $X$ is in $\mathscr{T}^{\prime}$ so is $X^{\prime}$. Thus we see that $\mathscr{T}^{\prime}$ is a torsion class for some torsion theory closed under taking submodules. Moreover, by property $D^{\prime}$ cited above $\mathscr{T}$ is contained in $\mathscr{T}^{\prime}$.

From the first part of the proof $\mathscr{T}$ and $\mathscr{T}^{\prime}$ are determined by their finitely generated modules. If $X^{\prime}$ is a finitely generated module not in $\mathscr{T}$ then $X^{\prime}=X_{\alpha}^{\prime}$ for some $\alpha$ and there exists a nonzero homomorphism $X^{\prime} \rightarrow F_{\alpha}=X_{\alpha}^{\prime} / T\left(X_{\alpha}^{\prime}\right)$. It follows that $\operatorname{Hom}_{R}\left(X^{\prime}, \pi F_{\alpha}\right) \neq 0$ and so $\operatorname{Hom}_{R}\left(X^{\prime}, Y_{0}\right) \neq 0$. Consequently $X^{\prime}$ is not in $\mathscr{T}^{\prime}$. Since $\mathscr{T}$ and $\mathscr{T}^{\prime}$ coincide on finitely generated modules, $\mathscr{T}=\mathscr{T}^{\prime}$.

From the properties $D$ and $D^{\prime}$ cited above the modules $X_{0}$ and $Y_{0}$ need not be unique because $X_{0} \oplus Y^{\prime}$ and $Y_{0} \oplus Y^{\prime}$ would do as well where $X^{\prime} \in \mathscr{T}$ and $Y^{\prime} \in \mathscr{F}$.

Before we develop a more specialized torsion theory in the next section, we should make a few remarks about torsion. In the first 
place a torsion theory $(\mathscr{T}, \mathscr{F})$ gives an idempotent subfunctor of the identity $T$, where $T$ applied to $M \stackrel{f}{\rightarrow} S$ gives $T(M) \stackrel{T(f)}{\rightarrow} T(S)$ and $T(f)$ is $f$ restricted to $T(M)$. Two torsion theories are the same if their torsion functors are equivalent.

If we apply this remark to the ring ${ }_{R} R$ considered as a left module over itself we see that the left ideal $T\left({ }_{R} R\right)$ is carried into itself by every right multiplication. That is, $T\left({ }_{R} R\right)$ is a two sided ideal. At first we thought that torsion theories could be indexed by these two sided ideals; that is, $T$ and $T^{\prime}$ are the same torsion functor if and only if $T\left({ }_{R} R\right)=T^{\prime}\left({ }_{R} R\right)$. However, the following example shows this is not correct: Let $\mathscr{T}^{\prime}$ be the torsion class containing only the zero module and let $\mathscr{T}_{0}=\left\{X \mid \operatorname{Hom}_{R}(X, Q(R))=0\right\}$ where $Q(R)$ is the minimal injective for $R$. These clearly give torsion theories; let $T_{0}$ and $T^{\prime}$ be the associated torsion functors. Then one sees that $T_{0}\left({ }_{R} R\right)=$ $0=T^{\prime}\left({ }_{R} R\right)$.

In $\S 3$ we shall investigate the torsion theory $\mathscr{T}_{0}$ defined above in more detail. Also we shall show in the next section that certain torsion theories can be indexed by ideals in the ring.

2. Torsion-torsion free classes. Let $\mathscr{T}$ be a class of modules in ${ }_{R}$ /l which is closed under taking submodules factors, extensions, direct products and direct sums. Then $\mathscr{T}$ is the torsion class for a torsion theory $(\mathscr{T}, \mathscr{F}$ ) closed under taking submodules and it is also the torsion free class for another torsion theory $(\mathscr{C}, \mathscr{T})$. Such a pair of torsion theories $(\mathscr{C}, \mathscr{T})$ and $(\mathscr{T}, \mathscr{F})$ will be called a torsiontorsion free theory (TTF theory) and the class $\mathscr{T}$, a TTF class.

Consider for a moment a class $\mathscr{T}$ closed under taking submodules, factors, extension and direct sum. Classes somewhat more general were considered by Gabriel [6, p. 395] as "closed" subcategories of ${ }_{R} \mathscr{C l}$. Such a class $\mathscr{T}$ is completely determined by the cyclic modules in it and therefore determines a filter $F_{T}$ of left ideals of $R[6, \mathrm{p}$. 411] where $L \in F_{T}$ if and only if $R / L \in T$. The filter $F_{T}$ is idempotent in the sense that the product of two left ideals in it is again in it [6, p. 412]. Conversely, any such idempotent filter determines such a class $\mathscr{T}[6$, p. 411-412].

The following theorem was mentioned by R. S. Pierce one day during a conversation.

THEOREM 2.1. The class $\mathscr{T}$ closed under taking submodules, factors, extension and direct sums is also closed under direct products if and only if its associated filter $F_{T}$ has a smallest element $I$. In that case $\mathscr{T}$ is a TTF class and $I$ is the two sided ideal $C(R)$ where $C$ is the torsion functor for the torsion theory $(\mathscr{C}, \mathscr{T})$. 
Proof. If $\mathscr{T}$ is closed under products the natural maps $R \rightarrow R / L$ for $R / L$ in $\mathscr{T}$ induce a map $R \stackrel{\theta}{\rightarrow} \prod_{R I L \in T} R / L$ and $\operatorname{Im} \theta$ is again in $\mathscr{T}$. But then $\operatorname{Ker} \theta$ is in the associated filter and we have $\operatorname{Ker} \theta \subseteq L$ for each $L$ in the associated filter and the filter therefore has a smallest element.

Conversely, suppose $I$ is the minimal element in the filter associated with $\mathscr{T}$. We shall show that $M$ is in $\mathscr{T}$ if and only if $I M=0$. If $M$ in $\mathscr{T}$ and $I M \neq 0$, then $I m \neq 0$ for some $m \in M$. The mapping: $I \rightarrow I m$ has image in $T$ so there exists $X \subset I$ such that $I / X=I m$. Now since $\mathscr{T}$ is closed under extension and the following exact sequence has both ends in $\mathscr{T}$

$$
0 \rightarrow I / X \rightarrow R / X \rightarrow R / I \rightarrow 0,
$$

it follows that $R / X$ is in $\mathscr{T}$. This latter statement contradicts the minimality of $I$.

Conversely, suppose that $I M=0$ and let $\Sigma R_{i}$ be a free $R$ module mapping onto $M, \Sigma R_{i} \stackrel{\theta}{\rightarrow} M \rightarrow 0$. Since $\operatorname{Ker} \theta$ contains $\Sigma I_{i}, \theta$ induces a map of $\Sigma R_{i} / I_{i}$ onto $M$. But since $\mathscr{T}$ is closed under direct sums and factors we have $M$ in $\mathscr{T}$.

Finally, it follows that $\mathscr{T}$ is closed under products because $I$ annihilates a product if and only if it annihilates each factor.

In the case that the filter has a smallest element $I$ then, $C(R)=I$ for $R / I$ is the "largest" factor of $R$ such that $I$ annihilates it.

We remark that the filter $F_{I}=\{L \mid L \supseteqq I, L$ a left ideal $\}$ will be an idempotent filter if and only if $I=I^{2}$. This idempotence is necessary for the class of modules annihilated by $I$ to be closed under extension (e.g. $0 \rightarrow I / I^{2} \rightarrow R / I^{2} \rightarrow R / I \rightarrow 0$ is a short exact sequence with $I$ annihilating each end but not the middle unless $I=I^{2}$ ).

COROLLARY 2.2. There is a one-to-one correspondence between TTF classes $T_{I}$ and idempotent two sided ideals $I$

$$
I \rightarrow\{M \mid I M=0\}=T_{I} .
$$

Proof. This follows from Gabriel's correspondence between "closed" categories of modules and idempotent filters of left ideals [6, p. 412] together with the above theorem which classifies those classes which are also closed under products.

COROLLARY 2.3. The following are equivalent for an ideal I

(1) $I=I^{2}$

(2) $T_{I}=\{M \mid I M=0\}$ is a TTF class

(3) the natural map $0 \rightarrow \operatorname{Ext}_{R / I}(A, C) \rightarrow \operatorname{Ext}_{R}(A, C)$ is an isomorphism for $R$ modules $A, C$ such that $I A=I C=0$. 
Proof. Only the condition (3) requires attention. For $R$ modules $A, C$ such that $I A=I C=0, R$ extensions can be considered as $R / I$ extensions because the class of modules annihilated by $I$ is closed under extension.

If $I \neq I^{2}$, by the remark preceding Corollary 2.2 ,

$$
0 \rightarrow I / I^{2} \rightarrow R / I^{2} \rightarrow R / I \rightarrow 0
$$

represents an $R$ extension in $\operatorname{Ext}_{R}\left(R / I, I / I^{2}\right)$ which is not an $R / I$ extension and in this case the map is not an isomorphism.

The following theorem gives information about a special class of TTF classes.

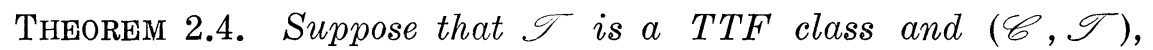
$(\mathscr{T}, \mathscr{F})$ the associated torsion theories with torsion functors $C, T$; then the following are equivalent.

(1) for all $M, M=C(M) \oplus T(M)$

(2) $\quad R=C(R) \oplus T(R)$ ring direct sum

(3) $\mathscr{H}=\mathscr{C}$

(4) $T(C(M))=0$ and $C(M / T(M))=M / T(M)$ for all $M$.

Proof. $\quad(1) \Longrightarrow(2)$ is trivial.

The class $\mathscr{T}$ is $\{M \mid C(R) M=0\}$. Let $\mathscr{A}$ be the class

$$
\{M \mid T(R) M=0\} \text {. }
$$

Assuming the decomposition $R=C(R) \oplus T(R)$ gives a decomposition $1=e_{1}+e_{2}$ where $e_{i}$ are orthogonal central idempotents in $R$. Then $\mathscr{T}$ and $\mathscr{A}$ can be characterized as $\mathscr{T}=\left\{M \mid e_{1} M=0\right\}$ and

$$
\left\{M \mid e_{2} M=0\right\}=\mathscr{A}
$$

and $e_{1}$ acts like identity on modules in $\mathscr{T}$. Using this one sees that $A$ can now be characterized as either

$$
\mathscr{A}=\left\{M \mid \operatorname{Hom}_{R}(M, T)=0 \text { all } T \text { in } \mathscr{T}\right\}
$$

or

$$
\mathscr{A}=\left\{M \mid \operatorname{Hom}_{R}(T, M)=0 \text { all } T \text { in } \mathscr{T}\right\} .
$$

It follows that $\mathscr{F}=\mathscr{C}$ and $(2 \Longrightarrow 3)$.

Assuming (3), $\mathscr{F}=\mathscr{C}$ is closed under sums, products, submodules, and factors, so $T(C(M))=0$ and since $M / T(M)$ in $\mathscr{F}, C(M / T(M))=$ $M / T(M)$ which is condition (4).

Finally, assuming (4), form $C(M) \cap T(M)$ for an $R$ module $M$. Since this is a submodule of $T(M)$ and $\mathscr{T}$ is closed under submodules, 
it is in $\mathscr{G}$. But $T(C(M))=0$ means that $C(M)$ has no $\mathscr{T}$ torsion submodules so $C(M) \cap T(M)=0$.

Now form $M /(C(M)+T(M))$, this is a factor of $M / C(M)$ which is in $\mathscr{T}$. It follows that $M /(C(M)+T(M))$ is in $\mathscr{T}$. Since we are assuming that $C(M / T(M))=M / T(M), M /(C(M)+T(M))$ is also in $\mathscr{C}$ since it is a factor of $M / T(M)$ which is in $\mathscr{C}$. But then

$$
M /(C(M)+T(M))
$$

is in both $\mathscr{C}$ and $\mathscr{T}$ and is therefore zero. Thus we have established that $M=C(M) \oplus T(M)$. This completes the proof of the theorem.

3. A particular torsion theory. In this section we define a torsion theory which coincides with the usual one for integral domains. This torsion has been used before [See Prop. 1 of 7].

Let $Q(R)$ be the injective hull of $R$ considered as a left module over itself [4]. Let $\mathscr{T}_{0}=\left\{M \mid \operatorname{Hom}_{R}(M, Q(R))=0\right\}$. It is not hard to show that $\mathscr{T}_{0}=\left\{M \mid \operatorname{Hom}_{R}\left(M^{\prime}, R\right)=0\right.$ for all submodules $M^{\prime} \subseteq M$. $\}$

The methods of $\S 1$ show that the class $\mathscr{T}_{0}$ is a torsion class closed under taking submodules. It is not, in general, closed under arbitrary direct products (e.g. $R=Z$ the ring of integers). However, in the following theorem we shall show that for a fairly wide class of rings $\mathscr{T}_{0}$ is a TTF class. Eilenberg [5] introduced the notation of a perfect ring and Bass [2] has given a characterization of them. We use Bass' characterization instead of Eilenberg's original definition.

Definition. $R$ is right perfect if

(1) $R / N$ is semisimple with minimum condition where $N$ is the Jacobson radical

(2) every nonzero left module $M$ has nonzero socle $S(M)$, where the socle is the submodule generated by the simple submodules of $M$.

Condition (2) is equivalent to " every nonzero module has simple submodules."

THEOREM 3.1. If $R$ is right perfect then $\mathscr{T}_{0}$ is a TTF class.

Proof. We must show that the product of $\mathscr{T}_{0}$-torsion modules is again $\mathscr{S}_{0}$-torsion.

Let us begin by defining for each ordinal $\alpha$ a submodule $S^{\alpha}(M)$ of the $R$ module $M$ as follows:

(1) $S^{0}(M)=0$

(2) if $\beta$ is a limit ordinal $S^{\beta}(M)=\bigcup_{\alpha<\beta} S^{\alpha}(M)$ 
(3) if $\beta=\alpha+1, S^{\beta}(M)$ is the inverse image of $S\left(M / S^{\alpha}(M)\right)$ under the map $M \rightarrow M / S^{\alpha}(M)$. Equivalently, $S^{\beta}(M)=\left\{m \mid N m \in S^{\alpha}(M)\right\}$ where $N$ is the radical of $R$.

From the assumptions on the ring $R$ we know that for some $\alpha_{0}$, $S^{\alpha_{0}}(M)=M$ (and the cardinal of that $\alpha_{0}$ is less than or equal to the cardinal of $M$ ). Also for $\alpha<\alpha_{0}$ we know that $S^{\alpha+1}(M) / S^{\alpha}(M)$ is a direct sum of simple $R$ (hence $R / N$ ) modules.

We now define the composition factors of the $R$ module $M$ to be those simple modules which appear as summands in $S^{\alpha+1}(M) / S^{\alpha}(M)$ for some ordinal $\alpha$. Note that since $R / N$ is semi-simple with minimum condition, $R$ has (up to isomorphism) only a finite set of simple modules and for each $M$ the composition factors of $M$ form a subset of that set.

Now we show that an $R$-module $M$ is $\mathscr{T}_{0}$-torsion if and only if for each composition factor $S$ of $M, \operatorname{Hom}_{R}(S, R)=0$. Suppose first that for each composition factor $S$ of $M, \operatorname{Hom}_{R}(S, R)=0$ but that $\operatorname{Hom}_{R}(M, Q(R)) \neq 0$. Let $f$ be a nonzero homomorphism of $M$ to $Q(R)$, since $R$ is essential in $Q(R), f(M) \cap R \neq 0$. Let $f^{-1}(f(M) \cap R)=M_{0}$ in $M$ and we have a nonzero homomorphism $f_{0}: M_{0} \rightarrow R$ where $f_{0}=$ $\left.f\right|_{M_{0}}$. Because $M_{0} \cap S^{\alpha_{0}}(M)=M_{0}$ we can be assured that there exists a first ordinal $\alpha_{1}$ such that $f_{0}$ restricted to $S^{\alpha_{1}}(M) \cap M_{0}$ is nonzero.

Now we note that $\alpha_{1}$ cannot be a limit ordinal, for if $S^{\alpha_{1}}(M)=$ $\bigcup_{\beta<\alpha_{1}} S^{\beta}(M)$ with $f_{0}$ restricted to $S^{\beta}(M) \cap M_{0}$, the zero map, then $f_{0}$ restricted to $S^{\alpha_{1}}(M) \cap M_{0}$ is also the zero map.

Let $\alpha_{1}=\gamma+1$ then $f_{0}$ induces a nonzero homomorphism of

$$
\left(S^{\gamma+1}(M) \cap M_{0}\right) /\left(S^{\gamma}(M) \cap M_{0}\right)
$$

into $R$. Since

$$
\left(S^{\gamma+1}(M) \cap M_{0}\right) /\left(S^{\gamma}(M) \cap M_{0}\right)
$$

is isomorphic to

$$
\left(S^{\gamma+1}(M) \cap M_{0}+S^{\gamma}(M)\right) / S^{\gamma}(M)
$$

we have a nonzero homomorphism of a submodule of $S^{\gamma+1}(M) / S^{\gamma}(M)$ into $R$. This gives a nonzero homomorphism of one of the composition factors of $M$ into contradicting the assumption that $\operatorname{Hom}_{R}(S, R) \neq 0$ for composition factors $S$ of $M$.

Conversely, suppose that $\operatorname{Hom}_{R}(S, R) \neq 0$ for some composition factor $S$ of $M$. This gives a nonzero homomorphism $f: M^{\prime} \rightarrow R$ where $M^{\prime}$ is a submodule of $M$ (actually $M^{\prime}$ can be taken to be $S^{\alpha+1}(M)$ where $S$ appears as a summand of $\left.S^{\alpha+1}(M) / S^{\alpha}(M)\right)$. Since $Q(R)$ is the 
injective hull of $R$ we have the solid diagram

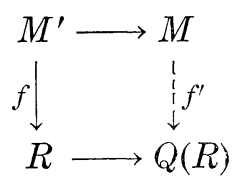

which can be extended by a dotted arrow $f^{\prime}$ which gives a nonzero element of $\operatorname{Hom}_{R}(M, Q(R))$. Thus $M$ is not $\mathscr{T}_{0}$-torsion.

Finally we complete the proof of the theorem by showing that the set of composition factors of a product $\prod_{i \in I} M_{i}$ is the union of the composition factors of each of the factors $M_{i}$. For each simple $R$ module $S_{i}$ there is an idempotent $\bar{e}_{i}$ in $R / N$ such that $\bar{e}_{i} S_{i} \neq 0$ and $\bar{e}_{i} S_{j}=0$ for $i \neq j$. Since $R$ is an $S B I$ ring [8, p. 53, 54] these can be raised to idempotents $e_{i}$ in $R$. Also we still have the relation $e_{i} S_{i} \neq 0$ and $e_{i} S_{j}=0$ for $i \neq j$.

Now we shall show that $S_{i}$ is a composition factor of $M$ if and only if $e_{i} M \neq 0$. First if $S_{i}$ is a composition factor of $M$ then $S_{i}$ appears as a summand of $S^{\alpha+1}(M)(M)$, for some ordinal $\alpha$. Thus, $e_{i} S^{\alpha+1}(M) \nsubseteq S^{\alpha}(M)$, and, a fortiori, $e_{i} M \neq 0$.

Conversely, if $e_{i} M \neq 0$, then $e_{i} m \neq 0$ for a suitable $m \in M$. There is a first ordinal $\alpha$ such that $e_{\imath} m \in S^{\alpha}(M)$. We claim that $\alpha=\gamma+1$ is not a limit ordinal for if $S^{\alpha}(M)=\bigcup_{\beta<\alpha} S^{\beta}(M)$ with $e_{i} m \notin S^{\beta}(M)$, then $\overline{e_{i} m} \notin S^{\alpha}(M)$ also. It follows that $e_{\imath} m$ represents a nonzero coset $\overline{e_{i} m}$ in $S^{\gamma+1}(M) / S^{\gamma}(M)$ and $e_{i} \overline{e_{i} m}=\overline{e_{i} m} \neq 0$. Therefore, one of the summands of $S^{\gamma+1}(M) / S^{\gamma}(M)$ is $S_{i}$ for otherwise $e_{i}$ would annihilate $S^{\gamma+1}(M) / S^{\gamma}(M)$. Thus we have shown that $S_{i}$ is a composition factor of $M$.

We now complete the proof of the theorem. It is clear that $e_{i}$ annihilates a product $\Pi_{j \in J} M_{j}$ if and only if $e_{i}$ annihilates each factor. So the set of composition factors of a product is the union of the composition factors of each of the factors. Thus for right perfect rings the product of $\mathscr{T}_{0}$-torsion modules is again $\mathscr{T}_{0}$-torsion.

The class of right perfect rings is fairly large, containing rings with minimum condition and semi-primary rings [2].

However, we shall show by an example that the rings for which $\mathscr{T}_{0}$-torsion is closed under products is even bigger.

Let $R=\prod_{i \in N} K_{i}$ the product of an infinite number of copies of a field. $R$ has an infinite set of orthogonal, indecomposable idempotents $\left\{e_{i}\right\}$. It isn't hard to show that a module $M$ is $\mathscr{T}_{0}$-torsion if and only if $e_{i} M=0$ for all $i$. It is clear that this property is inherited by products, so for such rings the product of $\mathscr{T}_{0}$ torsion is again $\mathscr{T}_{0}$ torsion. Note also that the ring $R$ has some non trivial torsion modules, for instance $\Pi K_{i} / \Sigma K_{i}$.

This brings up the question of the structure of rings for which 
(0) is the only $\mathscr{T}_{0}$ torsion module. Clearly, in this case, $\mathscr{T}_{0}$ torsion will be closed under products. The following theorem gives several equivalent characterizations of this condition. In a ring $R$ we denote by $r(S)$, the right annihilator of $S, r(S)=\{x \mid S x=0\}$.

THEOREM 3.2. For a ring $R$ the following conditions are equivalent:

(1) $\mathscr{T}_{0}=(0)$

(2) For every simple $R$-module $S, \operatorname{Hom}_{R}(S, R) \neq 0$

(3) For every maximal left ideal $L$ of $R, r(L) \neq 0$

(4) For every left ideal $L \neq R, r(L) \neq 0$

Proof. We prove the implications cyclically in order. If $\mathscr{T}_{0}=0$ then $\operatorname{Hom}_{R}(S, Q(R)) \neq 0$ for simple modules $S$. But if $f: S \rightarrow Q(R)$ is nonzero, then $f(S) \cap R \neq 0$ and it follows that $f(S) \subseteq R$ since $f(S)$ is also simple. Therefore $\operatorname{Hom}_{R}(S, R) \neq 0$ and (1) implies (2).

Now let $L$ be a maximal left ideal of $R$ and consider the sequence $0 \rightarrow L \rightarrow R \rightarrow R / L \rightarrow 0$. Note that $R / L$ is simple. The sequence induces $0 \rightarrow \operatorname{Hom}_{R}(R / L, R) \rightarrow \operatorname{Hom}_{R}(R, R)$ exact. Considering $\operatorname{Hom}_{R}(R / L, R)$ as a submodule of $\operatorname{Hom}_{R}(R, R)$, the right multiplications of $R$ we see that $\operatorname{Hom}_{R}(R / L, R)=r(L)$ the right annihilator of $L$. It follows that $r(L) \neq 0$ because $\operatorname{Hom}_{R}(R / L, R) \neq 0$.

Since every left ideal $L \neq R$ can be embedded in a maximal one $\bar{L}$ and the relation $L \leqq \bar{L}$ implies $r(\bar{L}) \subseteq r(L)$, it follows that (3) implies (4).

Finally, if for each $L \neq R, r(L) \neq 0$, then for each such $L$, $\operatorname{Hom}_{R}(R / L, R) \neq 0$ (a right multiplication by a nonzero element of $r(L)$ gives such a nonzero homomorphism). It follows that

$$
\operatorname{Hom}_{R}(R / L, Q(R)) \neq 0
$$

for all $L \neq R$ and the filter of left ideals associated with $\mathscr{T}_{0}$ in this case consists only of $R$ itself. That is, $C(R)=R$. Then from Theorem 2.1 we know that $\mathscr{T}_{0}=(0)$. This completes the proof of the theorem.

Remark. M. Auslander has shown [1] that a commutative semiprimary ring satisfies condition (2) of the above theorem. Hence such rings have only zero torsion.

The following example shows that left and right torsion theories need not give rise to the same two sided ideal. Let

$$
R=\left\{\left(\begin{array}{ll}
x & 0 \\
y & z
\end{array}\right) \mid x, y, z \in K, \text { a field }\right\} \text {. }
$$

The left $\mathscr{T}_{0}$ torsion modules for $R$ are those annihilated by 


$$
C(R)=\left\{\left(\begin{array}{ll}
0 & 0 \\
y & z
\end{array}\right) \mid y, z \in K\right\},
$$

whereas the right $\mathscr{T}_{0}$ torsion modules are those annihilated by the ideal $C_{r}(R)=\left\{\left(\begin{array}{ll}x & 0 \\ y & 0\end{array}\right) \mid x, y \in K\right\}$.

\section{REFERENCES}

1. M. Auslander, On the dimension of modules and algebras III, Nagoya Math. J. 9 (1955), 67-77.

2. H. Bass, Finitistic Dimension and a homological generalization of semi primary rings, Trans. Amer. Math. Soc. 95 (1960), 466-488.

3. S. E. Dickson, A torsion theory for abelian categories, (to appear).

4. B. Eckmann, and Schopf, Über injective Moduln, Archiv der Math. 4 (1953), 7578.

5. S. Eilenberg, Homological dimension and Syzygies, Ann. of Math. 64 (1956), 328336.

6. P. Gabriel, Des Categories Abeliennes, Bull. Soc. Math. France, 90 (1962), 323-448.

7. E. Gentile, Singular submodule and injective hull, Indagationes Math. 24 (1962), 426-433.

8. N. Jacobson, Structure of Rings, Amer. Math. Soc. Colloquium Publications, 37 (1956).

9. J. P. Jans, Rings and Homology, Holt Rinehart and Winston, New York (1964). 10. C. Walker, and E. Walker, Quotient Categories and Rings of Quotients, (to appear). 



\title{
PACIFIC JOURNAL OF MATHEMATICS
}

\author{
EDITORS
}

\author{
H. SAmelson \\ Stanford University \\ Stanford, California \\ R. M. Blumenthal \\ University of Washington \\ Seattle, Washington 98105
}

\author{
J. DugundjI \\ University of Southern California \\ Los Angeles, California 90007
}

*Richard Arens

University of California

Los Angeles, California 90024

\section{ASSOCIATE EDITORS}
E. F. BECKENBACH
B. H. NeUmanN
F. WOLF
K. YoSIDA

\section{SUPPORTING INSTITUTIONS}

\author{
UNIVERSITY OF BRITISH COLUMBIA \\ CALIFORNIA INSTITUTE OF TECHNOLOGY \\ UNIVERSITY OF CALIFORNIA \\ MONTANA STATE UNIVERSITY \\ UNIVERSITY OF NEVADA \\ NEW MEXICO STATE UNIVERSITY \\ OREGON STATE UNIVERSITY \\ UNIVERSITY OF OREGON \\ OSAKA UNIVERSITY \\ UNIVERSITY OF SOUTHERN CALIFORNIA
}

\author{
STANFORD UNIVERSITY \\ UNIVERSITY OF TOKYO \\ UNIVERSITY OF UTAH \\ WASHINGTON STATE UNIVERSITY \\ UNIVERSITY OF WASHINGTON \\ * * * * \\ AMERICAN MATHEMATICAL SOCIETY \\ CALIFORNIA RESEARCH CORPORATION \\ SPACE TECHNOLOGY LABORATORIES \\ NAVAL ORDNANCE TEST STATION
}

Mathematical papers intended for publication in the Pacific Journal of Mathematics should by typewritten (double spaced). The first paragraph or two must be capable of being used separately as a synopsis of the entire paper. It should not contain references to the bibliography. No separate author's resumé is required. Manuscripts may be sent to any one of the four editors. All other communications to the editors should be addressed to the managing editor, Richard Arens, at the University of California, Los Angeles, California 90024.

50 reprints per author of each article are furnished free of charge; additional copies may be obtained at cost in multiples of 50 .

The Pacific Journal of Mathematics is published quarterly, in March, June, September, and December. Effective with Volume 13 the price per volume (4 numbers) is $\$ 18.00$; single issues, $\$ 5.00$. Special price for current issues to individual faculty members of supporting institutions and to individual members of the American Mathematical Society: $\$ 8.00$ per volume; single issues $\$ 2.50$. Back numbers are available.

Subscriptions, orders for back numbers, and changes of address should be sent to Pacific Journal of Mathematics, 103 Highland Boulevard, Berkeley 8, California.

Printed at Kokusai Bunken Insatsusha (International Academic Printing Co., Ltd.), No. 6, 2-chome, Fujimi-cho, Chiyoda-ku, Tokyo, Japan.

PUBLISHED BY PACIFIC JOURNAL OF MATHEMATICS, A NON-PROFIT CORPORATION

The Supporting Institutions listed above contribute to the cost of publication of this Journal, but they are not owners or publishers and have no responsibility for its content or policies.

* Basil Gordon, Acting Managing Editor until February 1, 1966. 


\section{Pacific Journal of Mathematics}

\section{Vol. 15, No. $4 \quad$ December, 1965}

Robert James Blattner, Group extension representations and the structure space ........... 1101

Glen Eugene Bredon, On the continuous image of a singular chain complex .............. 1115

David Hilding Carlson, On real eigenvalues of complex matrices .................... 1119

Hsin Chu, Fixed points in a transformation group ............................. 1131

Howard Benton Curtis, Jr., The uniformizing function for certain simply connected Riemann

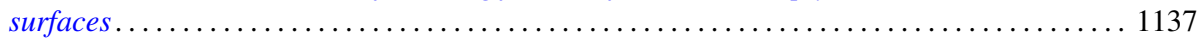

George Wesley Day, Free complete extensions of Boolean algebras................... 1145

Edward George Effros, The Borel space of von Neumann algebras on a separable Hilbert

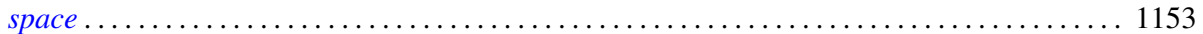

Michel Mendès France, $A$ set of nonnormal numbers ......................... 1165

Jack L. Goldberg, Polynomials orthogonal over a denumerable set ................ 1171

Frederick Paul Greenleaf, Norm decreasing homomorphisms of group algebras . . . . . . . . 1187

Fletcher Gross, The 2-length of a finite solvable group ........................ 1221

Kenneth Myron Hoffman and Arlan Bruce Ramsay, Algebras of bounded sequences ........ 1239

James Patrick Jans, Some aspects of torsion . . . . . . . . . . . . . . . . . . . . . . . 1249

Laura Ketchum Kodama, Boundary measures of analytic differentials and uniform

approximation on a Riemann surface ............................... 1261

Alan G. Konheim and Benjamin Weiss, Functions which operate on characteristic

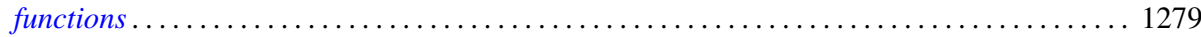

Ronald John Larsen, Almost invariant measures ............................ 1295

You-Feng Lin, Generalized character semigroups: The Schwarz decomposition ............ 1307

Justin Thomas Lloyd, Representations of lattice-ordered groups having a basis . . . . . . . . 1313

Thomas Graham McLaughlin, On relative coimmunity ....................... 1319

Mitsuru Nakai, $\Phi$-bounded harmonic functions and classification of Riemann surfaces ....... 1329

L. G. Novoa, On n-ordered sets and order completeness ..................... 1337

Fredos Papangelou, Some considerations on convergence in abelian lattice-groups . . . . . . . 1347

Frank Albert Raymond, Some remarks on the coefficients used in the theory of homology

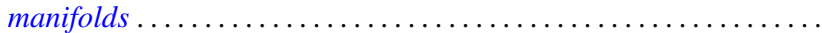

John R. Ringrose, On sub-algebras of a $C^{*}$-algebra .

Jack Max Robertson, Some topological properties of certain spaces of differentiable

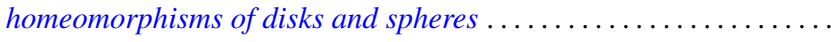

Zalman Rubinstein, Some results in the location of zeros of polynomials

Arthur Argyle Sagle, On simple algebras obtained from homogeneous general Lie triple systems. . . .

Hans Samelson, On small maps of manifolds ............................... 1401

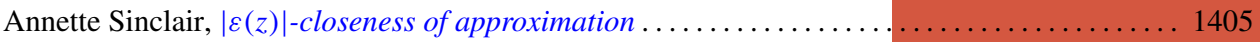

Edsel Ford Stiel, Isometric immersions of manifolds of nonnegative constant sectional curvature

Earl J. Taft, Invariant splitting in Jordan and alternative algebras ................. 1421

L. E. Ward, On a conjecture of R. J. Koch . . . . . . . . . . . . . . . . . . . . . . . . . . . 1429

Neil Marchand Wigley, Development of the mapping function at a corner . . . . . . . . . . 1435

Horace C. Wiser, Embedding a circle of trees in the plane ....................... 1463

Adil Mohamed Yaqub, Ring-logics and residue class rings . . . . . . . . . . . . . . . . 1465

John W. Lamperti and Patrick Colonel Suppes, Correction to: Chains of infinite order and their application to learning theory ........................................ 1471

Charles Vernon Coffman, Correction to: Non-linear differential equations on cones in Banach

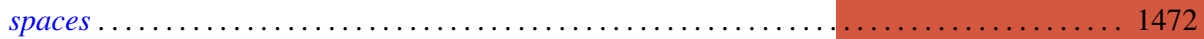

P. H. Doyle, III, Correction to: A sufficient condition that an $\operatorname{arc}$ in $S^{n}$ be cellular. . . . . . . . 1474

P. P. Saworotnow, Correction to: On continuity of multiplication in a complemented algebra 